\title{
Space weather and dangerous phenomena on the Earth: principles of great geomagnetic storms forcasting by online cosmic ray data
}

\author{
L. I. Dorman ${ }^{1,2}$ \\ ${ }^{1}$ Israel Cosmic Ray and Space Weather Center and Emilio Segre' Observatory, affiliated to Tel Aviv University, Technion and \\ Israel Space Agency, P. O. Box 2217, Qazrin 12900, Israel \\ ${ }^{2}$ Cosmic Ray Department of IZMIRAN, Russian Academy of Science, Troitsk 142092, Moscow Region, Russia
}

Received: 25 February 2005 - Revised: 24 May 2005 - Accepted: 28 May 2005 - Published: 22 November 2005

Part of Special Issue "1st European Space Weather Week (ESWW)"

\begin{abstract}
According to NOAA space weather scales, geomagnetic storms of scales G5 (3-h index of geomagnetic activity $\left.K_{p}=9\right), \mathrm{G} 4\left(K_{p}=8\right)$ and $\mathrm{G} 3\left(K_{p}=7\right)$ are dangerous for satellites, aircrafts, and even for technology on the ground (influence on power systems, on spacecraft operations, on $\mathrm{HF}$ radio-communications and others). We show on the basis of statistical data, that these geomagnetic storms, mostly accompanied by cosmic ray (CR) Forbush-decreases, are also dangerous for people's health on spacecraft and on the ground (increasing the rate of myocardial infarctions, brain strokes and car accident road traumas). To prevent these serious damages it is very important to forecast dangerous geomagnetic storms. Here we consider the principles of using CR measurements for this aim: to forecast at least 10$15 \mathrm{~h}$ before the sudden commencement of great geomagnetic storms accompanied by Forbush-decreases, by using neutron monitor muon telescope worldwide network online hourly data. We show that for this forecast one may use the following features of $\mathrm{CR}$ intensity variations connected with geomagnetic storms accompanied by Forbush-decreases: 1) CR pre-increase, 2) CR pre-decrease, 3) CR fluctuations, 4) change in the 3-D CR anisotropy.
\end{abstract}

Keywords. Magnetic storms (Negative effects on the Earth's environment; Cosmic rays; Forbush-decrease and precursory effects; Method of forecasting)

1 On the influence of geomagnetic storms accompanied by cosmic ray Forbush-decreases on people's health and technology

There are numerous indications that natural, solar variabilitydriven time variations of the Earth's magnetic field can be

Correspondence to: L. I. Dorman

(lid@physics.tecnion.ac.il) hazardous to human health and safety. There are two lines of possible influence: effects on physical systems and on human beings as biological systems. High frequency radio communications are disrupted, electric power distribution grids are blacked out when geomagnetic-induced currents cause safety devices to trip, and atmospheric warming causes increased drag on satellites. An example of a major disruption on high technology operations by magnetic variations of a large extent occurred in March 1989, when an intense geomagnetic storm upset communication systems, orbiting satellites, and electric power systems around the world. Several large power transformers also failed in Canada and the United States, and there were hundred disoperation of relays and protective systems (Kappenman and Albertson, 1990; Hruska and Shea, 1993). Some evidence has been also reported on the association between geomagnetic disturbances and increases in work and traffic accidents (Ptitsyna et al., 1998 and references therein). These studies were based on the hypothesis that a significant part of traffic accidents could be caused by the incorrect or retarded reaction of drivers to the traffic circumstances, the capability to react correctly being influenced by the environmental magnetic and electric fields. The analysis of accidents caused by human factors in the biggest atomic station of the former USSR, "Kurskaya", during 1985-1989, showed that $\sim 70 \%$ of these accidents happened on the days of geomagnetic storms. In Reiter (1955) it was found that work and traffic accidents in Germany were associated with disturbances in atmospheric electricity and in geomagnetic field (defined by sudden perturbations in radio-wave propagation). On the basis of 25 reaction tests, it was also found that the human reaction time, during these disturbed periods, was considerably retarded. Retarded reaction in connection with naturally occurring magnetic field disturbances was also observed by König and Ankermüller (1982). 


\begin{tabular}{|c|c|c|c|}
\hline \multicolumn{2}{|c|}{$\begin{array}{c}\text { Geomagnetic } \\
\text { Storms }\end{array}$} & \multirow[b]{2}{*}{$\begin{array}{l}\text { Effects } \\
\text { Biological effects: increasing more than } 10-15 \% \text { of the daily rate of } \\
\text { myocardial infarction, brain strokes and car road accident traumas for } \\
\text { the population on the ground. } \\
\text { Power systems: widespread voltage control problems and protective } \\
\text { system problems can occur, some grid systems may experience a } \\
\text { complete collapse or blackouts. Transformers may experience } \\
\text { damage. } \\
\text { Spacecraft operations: may experience extensive surface charging, } \\
\text { problems with orientation, uplink/downlink and tracking satellites. } \\
\text { Other systems: pipeline currents can reach hundreds of amps, HF } \\
\text { (high frequency) radio propagation may be impossible in many areas } \\
\text { for one to two days, satellite navigation may be degraded for days, } \\
\text { low-frequency radio navigation can be out for hours. }\end{array}$} & \multirow{2}{*}{$\begin{array}{c}\begin{array}{c}\text { Number } \\
\text { per solar } \\
\text { cycle }\end{array} \\
4 \text { per } \\
\text { cycle } \\
\text { (4 days } \\
\text { per cycle) }\end{array}$} \\
\hline G5 & Extreme & & \\
\hline G4 & $\begin{array}{l}\text { Severe } \\
\text { Kp }=8\end{array}$ & $\begin{array}{l}\text { Biological effects: increasing several percent (up to } 10-15 \% \text { ) of the } \\
\text { daily rate of myocardial infarction, brain strokes and car road accident } \\
\text { traumas for the population on the ground. } \\
\text { Power systems: possible widespread voltage control problems and } \\
\text { some protective systems will mistakenly trip out key assets from the } \\
\text { grid. } \\
\text { Spacecraft operations: may experience surface charging and } \\
\text { tracking problems, corrections may be needed for orientation } \\
\text { problems. } \\
\text { Other systems: induced pipeline currents affect preventive measures, } \\
\text { HF radio propagation sporadic, satellite navigation degraded for } \\
\text { hours, low-frequency radio navigation disrupted. }\end{array}$ & $\begin{array}{c}100 \text { per } \\
\text { cycle } \\
\text { (60 days } \\
\text { per cycle) }\end{array}$ \\
\hline G3 & $\begin{array}{l}\text { Strong } \\
\text { Kp }=7\end{array}$ & $\begin{array}{l}\text { Biological effects: increasing a few percent of the daily rate of } \\
\text { myocardial infarction, brain strokes and car road accident traumas for } \\
\text { the population on the ground. } \\
\text { Power systems: voltage corrections may be required, false alarms } \\
\text { triggered on some protection devices. } \\
\text { Spacecraft operations: surface charging may occur on satellite } \\
\text { components, drag may increase on low-Earth-orbit satellites, and } \\
\text { corrections may be needed for orientation problems. } \\
\text { Other systems: intermittent satellite navigation and low-frequency } \\
\text { radio navigation problems may occur, HF radio may be intermittent. }\end{array}$ & $\begin{array}{c}200 \text { per } \\
\text { cycle } \\
\text { (130 days } \\
\text { per cycle) }\end{array}$ \\
\hline
\end{tabular}

Table 1. The extended NOAA scale of geomagnetic storms' influence on people's health, power systems, on spacecraft operations, and on other systems (greatest three types, mostly accompanied by Forbush-decreases); in the original NOAA scale we include biological effects, according to results discussed above.

Moreover, a number of investigations showed a significant correlation between the incidence of clinically important pathologies and strong geomagnetic field variations. The most significant results have been those on cardiovascular and nervous system diseases, showing some association with geomagnetic activity; a number of laboratory results on the correlation between human blood system, and solar and geomagnetic activity supported these findings (Ptitsyna et al., 1998 and references therein). Recently, the monitoring of the cardiovascular function among cosmonauts of the "MIR" space station revealed a reduction in heart rate variability during geomagnetic storms (Baevsky et al., 1997); the reduction in heart rate variability has been associated with a $550 \%$ increase in the risk of coronary artery diseases (Baevsky et al., 1997 and references therein). On the basis of statistical data on several million medical events in Moscow and in
St. Petersburg a sufficient influence of geomagnetic storms was found, accompanied by CR Forbush-decreases on the frequency of myocardial infarctions, brain strokes and car accident road traumas (Villoresi et al., 1994, 1995). Earlier we found that among all characteristics of geomagnetic activity, Forbush decreases are better related to hazardous effects of solar variability-driven disturbances of the geomagnetic field (Ptitsyna et al., 1998). Figure 1 shows the correlation between cardiovascular diseases, car accidents and different characteristics of geomagnetic activity (planetary index AA, major geomagnetic storms MGS, sudden commencement of geomagnetic storm SSC, occurrence of a downward vertical component of the interplanetary magnetic field $B_{z}$ and also the CR intensity decreasing phase of Forbush decreases (FD)). 

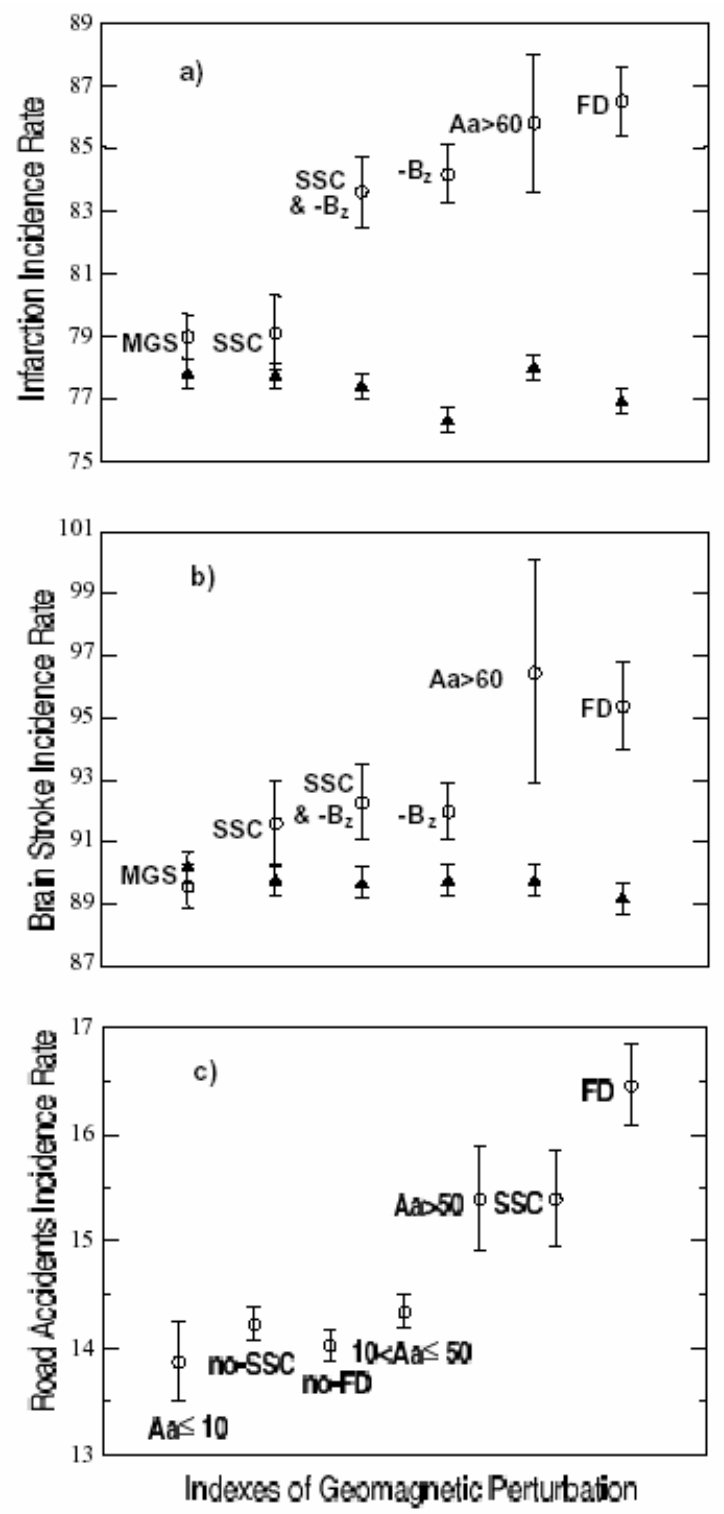

Fig. 1. Myocardial infarction (a), brain stroke (b), and road accident (c) incidence rates per day during geomagnetic quiet and perturbed days, according to different indexes of activity.

The most remarkable and statistically significant effects have been observed during days of geomagnetic perturbations, defined by the days of the declining phase of FD in $\mathrm{CR}$ intensity. During these days, the average number of traffic accidents, infarctions, and brain strokes increase by $(17.4 \pm 3.1) \%,(10.5 \pm 1.2) \%$ and $(7.0 \pm 1.7) \%$, respectively. In Fig. 2 we show the effect on pathology rates during the time development of FD. All FD have been divided into two groups, according to the time duration $\mathrm{T}$ of the FD decreasing phase. Then, the average incidence of infarctions and traffic accidents was computed, beginning one day before the FD-onset, until 5 days after. For the first group $(T<1$ day) the average daily incidences of infarctions and traffic incidence increases only in the first day of FD; no effect is ob-
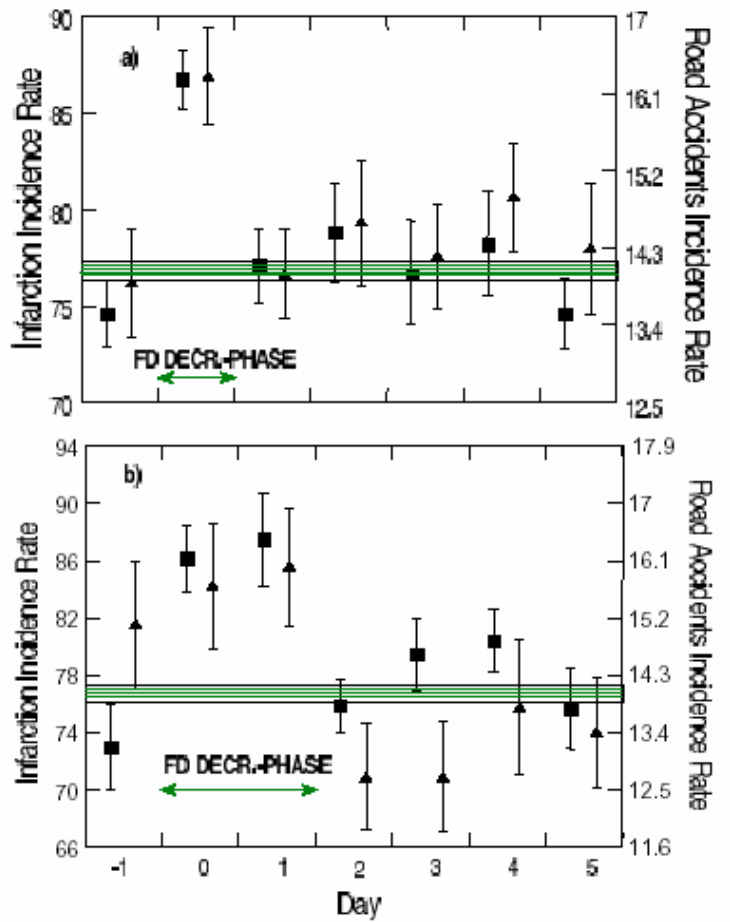

Fig. 2. Infarction (full squares) and road accident (full triangles) incidence during the time development of an FD: (a) CR decrease phase $\mathrm{T}<1$ day; (b) $\mathrm{CR}$ decrease phase 1 day $<\mathrm{T}<2$ days. With horizontal lines are shown average values for days without FD.

served during the recovery phase (that usually lasts for several days). Also, for the second group ( 1 day $<\mathrm{T}<2$ days) the increase in incidence rates is observed only during the 2-day period of the decreasing phase of FD. In Table 1 the NOAA scale of geomagnetic storm influence on power systems, on spacecraft operations, and on other systems (greatest three types, mostly accompanied by Forbush-decreases) is shown. We expect that for these three types of geomagnetic storms, online one-hour CR data of neutron monitors and muon telescopes for online forecasting (at least before 15-20 h of SSC) can be useful. In Table 1 we added some preliminary information on possible biological effects, according to our results discussed above (see Figs. 1 and 2).

\section{Using online one-hour cosmic ray data for forecast- ing dangerous geomagnetic storms accompanied by Forbush-decreases}

FD events can thus be used as reliable indicators of dangerous geomagnetic storms. The scheme of CR processes in the interplanetary space, that can be used for forecasting, is shown in Fig. 3. For a practical realization of forecasting hazardous geomagnetic storms by means of FD indicators, it will be necessary to obtain data from most CR stations in real time (currently most of the data are available only after about one month, and only about 10 stations provide online data through their Web sites on the Internet). Therefore, 


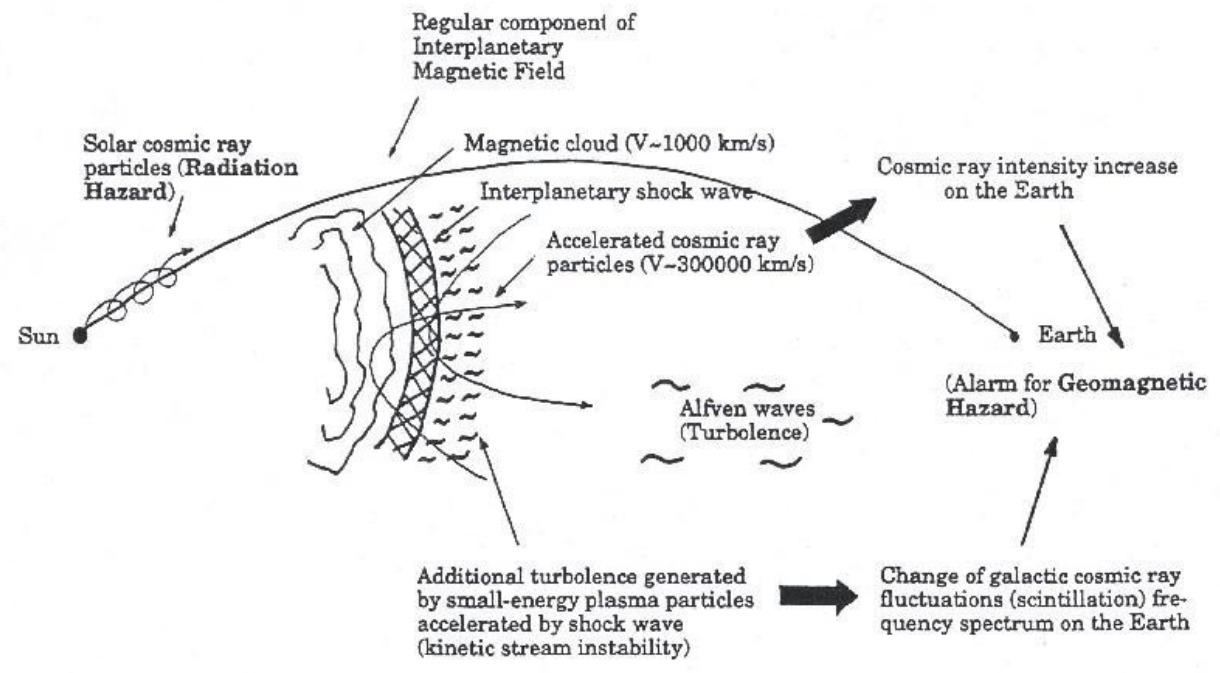

Fig. 3. The scheme of possible precursory effects in CR.

it is necessary to found a special Real-Time Cosmic Ray World Data Center to transform the cosmic ray station network into a real-time working International Cosmic Ray Service (ICRS) (Dorman, 1993; Dorman et al., 1993a,b,c). In these papers basic ideas of the organization of such realtime data collection and processing for providing a reliable forecast-service of FD and related dangerous disturbances of geomagnetic field were presented. According to Fig. 3, the main features observed in $\mathrm{CR}$ intensity variations before the beginning of great geomagnetic storms, caused by interplanetary shock waves and accompanied by FD that can be used for forecasting are the following (Dorman et al., 1995a,b, 1997, 1999; Munakata et al., 2000; in detail it was considered in Dorman, 2004):

1. CR pre-increase: (Blokh et al., 1959; Dorman, 1959; see review in Dorman, 1963a,b). The discovery of this effect in 1959 (Blokh et al., 1959) stimulated the development of the mechanism of galactic CR interactions with interplanetary shock waves (Dorman, 1959; Dorman and Freidman, 1959) and further analyses (Dorman et al., 1995a,b; Belov et al., 1995a,b) showing that this effect is related to particle interaction and acceleration by interplanetary shock waves;

2. CR pre-decrease: (McCracken and Parsons, 1958; Fenton et al., 1959; see review in Dorman, 1963a,b). This effect was analyzed recently, both theoretically (Dorman et al., 1995) and experimentally on the basis of the network of CR stations (Belov et al., 1995a,b). The predecrease effect can be due to a magnetic connection of the Earth with regions (moving from the Sun) with reduced CR density; this lower density can be observed at the Earth along the actual direction of IMF lines (Nagashima et al., 1990; Bavassano et al., 1994);

3. CR fluctuations: Many authors found some peculiarities in the behavior of CR fluctuations before FD: changes in frequency spectrum; appearance of peaks in spectrum at some frequencies; variations in some special parameter introduced for characterizing the variability of fluctuations. Though the obtained results are often contradictory (Dorman et al., 1995b), sometimes CR fluctuations appear as reliable phenomena for FD prediction, as expected from additional Alfvén turbulence produced by kinetic stream instability of low-energy particles accelerated by shock waves (Berezhko et al., 1997);

4. Change in 3-D CR anisotropy: The CR longitudinal dependence changes abruptly in direction close to the usual directions of the interplanetary magnetic field and depends on the character and source of the disturbance (Belov et al., 1995b, Dorman et al., 1995a,b). These effects, appearing much before Forbush decreases (up to 1 day), may be considered as predictors of FD. An estimation of the CR anisotropy vector may be done by the global survey method shortly described in Belov et al. (1997), and in detail in Chapter 3 of Dorman (2004).

\section{Example of cosmic ray precursory effects: asymptotic longitude - universal time cosmic ray intensity distri- bution}

In Fig. 4 we show an example of such an estimation done for the geomagnetic storm of 9 September 1992, representing a asymptotic longitude-universal time CR intensity distribution. In this figure a CR intensity pre-increase (yellow circles) and pre-decrease and Forbush-decrease (red circles), before and after the Sudden Storm Commencement (SSC) of great magnetic storm on 9 September 1992 are shown. The bigger diameter of the circle means a bigger amplitude of $\mathrm{CR}$ intensity variation. The vertical line marks the time of the SSC. From Fig. 4 it can be seen that the pre-increase, as 


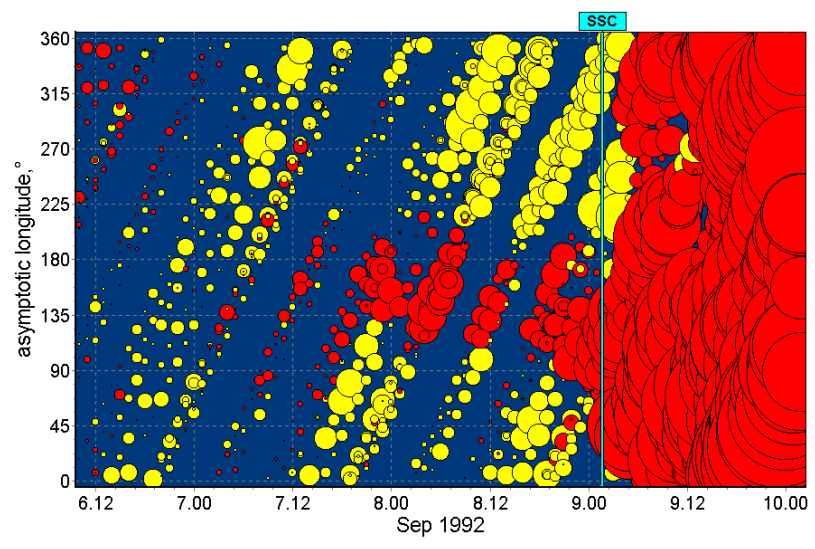

Fig. 4. Galactic CR intensity pre-increase (yellow circles), and predecrease and Forbush - decrease (red circles) before and after the Sudden Storm Commencement (SSC) of the great magnetic storm on 9 September 1992. The bigger diameter of circle means a bigger amplitude of CR intensity variation.

well as the pre-decrease, occurs $15-20 \mathrm{~h}$ before the SSC of the geomagnetic storm.

\section{Conclusions}

From the results discussed above it follows that the strong geomagnetic storm accompanied by a Forbush-decrease in $\mathrm{CR}$ intensity has clear pre-cursor effects which can be used for forecasting about $10-15 \mathrm{~h}$ before a SSC on a geomagnetic storm. The results obtained are in agreement with those obtained previously by Belov et al. (1995a,b); Dorman et al. (1995, 1999); Munakata et al. (2000). As it was shown by Munakata et al. (2000), the CR pre-increase and pre-decrease effects can be also observed very clearly by the multi-directional muon telescope world network. They investigated 14 "major" geomagnetic storms characterized by $K_{p} \geq 8-$ and 25 large storms characterized by $K_{p} \geq 7-$ observed in 1992-1998. It was shown that $89 \%$ of the "major" geomagnetic storms have clear precursor effects, which can be used for forecasting (the probability of exact forecasting increases with increasing storm strength). We suggest that the analysis described above, on the basis of online one-hour neutron monitor and muon telescope data from the world network of CR stations and observatories, can be made in the near future automatically for forecasting great geomagnetic storms. This important problem can be solved, for example, by the International Cosmic Ray Service (proposed in Dorman, 1993; Dorman et al., 1993a,b,c) that will be based on real-time collection and exchange through the Internet or FTP of the data from all cosmic-ray stations of the network. Some examples of such analysis in historical events are given in (Dorman et al. (2003)).

Acknowledgements. Our great thanks to Y. Ne'eman and A. Sternlieb for constant interest and support of the work of Israel Cosmic Ray and Space Weather Center and Emilio Segre' Observatory, and
J. Allen, A. V. Belov, E. A. Eroshenko, N. Iucci, J. Lilensten, K. G. McCracken, M. Murat, M. Parisi, L. Pustil'nik, M. A. Shea, D. F. Smart, M. Storini, G. Villoresi, V. G. Yanke, and I. G. Zukerman for collaboration and interesting discussions. Our thanks to both Reviewers for useful comments. The work of neutron monitor in Emilio Segre' Observatory on Mt. Hermon is supported by Tel Aviv University, UNIRoma-Tre, and IFSI-CNR Collaboration. This research is partly supported by the European Project COST-724.

Topical Editor T. Pulkkinen thanks two referees for their help in evaluating this paper.

\section{References}

Baevsky, R. M., Petrov, V. M., and Cornélissen, G., et al.: Metaanalyzed heart rate variability, exposure to geomagnetic storms, and the risk of ischemic heart disease, Scripta medica, 70, 199 204, 1997.

Bavassano, B., Iucci, N., Lepping, R. P., Signorini, C., Smith, E. J., and Villoresi, G.: Galactic cosmic ray modulation and interplanetary medium perturbations due to a long-living active region during October 1989, J. Geophys Res., 99, 4227-4234, 1994.

Belov, A. V., Dorman, L. I., Eroshenko, E. A., Iucci, N., Villoresi, G., and Yanke, V. G.: Search for predictors of Forbush-decreases, Proc. 24th Intern. Cosmic Ray Conf., Rome, 4, 888-891, 1995a.

Belov, A. V., Dorman, L. I., Eroshenko, E. A., Iucci, N., Villoresi, G., and Yanke, V. G.: Anisotropy of cosmic rays and Forbushdecreases in 1991, Proc. 24th Intern. Cosmic Ray Conf., Rome, 4, 912-915, 1995b.

Belov, A. V., Eroshenko, E. A., and Yanke, V. G.: Modulation effects in 1991-1992 years, Proc. 25th Intern. Cosmic Ray Conf., Durban, 1, 437-440, 1997.

Berezhko, E. B., Kozlov, V. I., Kuzmin, A. I., and Tugolukov, N. N.: Cosmic ray intensity micropulsations assotiated with disturbances of electromagnetic conditions in the Heliosphere, Proc. 20th Intern. Cosmic Ray Conf., Moscow, 4, 99-102, 1987.

Blokh, Ya. L., Glokova, E. S., Dorman, L. I., and Inozemtseva, O. I.: Electromagnetic conditions in interplanetary space in the period from 29 August to 10 September 1957 determined by cosmic ray variation data, Proc. 6th Intern. Cosmic Ray Conf., Moscow, 4, 172-177, 1959.

Dorman, L. I.: On the energetic spectrum and lengthy of cosmic ray intensity increase on the Earth caused by shock wave and albedo from magnetized front of corpuscular stream, Proc. 6th Intern. Cosmic Ray Conf., Moscow, 4, 132-139, 1959.

Dorman, L. I.: Cosmic Ray Variations and Space Research, Nauka, Moscow, 1963a.

Dorman, L. I.: Geophysical and Astrophysical Aspects of Cosmic Rays, North-Holland Publ. Co., Amsterdam, in: Progress in Physics of Cosmic Ray and Elementary Particles, (Eds.) Wilson, J. G. and Wouthuysen, S. A.), 7, 1963b.

Dorman, L. I.: Cosmic Rays: Variations and Space Exploration, North-Holland, Amsterdam, 1974.

Dorman, L. I.: On the Cosmic Ray World Service, Izvestia Academy of Science of USSR, Ser. Phys., 57, 7, 149-152, 1993.

Dorman, L. I.: Solar energetic particle events and geomagnetic storms influence on people's health and technology; Principles of monitoring and forecasting of space dangerous phenomena by using online cosmic ray data, Proc. 22nd, in: ISTC Japan Workshop on Space Weather Forecast in Russia/CIS, (Ed.) Muraki, Y., Nagoya University, 2, 133-151, 2002. 
Dorman, L. I.: Cosmic rays and Space Weather, The Early Universe and the Cosmic Microwave Background: Theory and Observations (Eds.) Sanchez, N. and Parijskij, Y. N., Kluwer Ac. Publishers, NATO Science Series, 130, 517-557, 2003.

Dorman, L. I.: Cosmic Rays in the Earth's Atmosphere and Underground, Kluwer Acad. Publishers, Dordrecht/Boston/London, 2004.

Dorman, L. I. and Freidman, G. I.: On the possibility of charged particle acceleration by shock waves in the magnetized plasma, Proc. of All-Union Conf. on Magneto-Hydrodynamics and Plasma Physics, Latvia SSR Academy of Sciences Press, Riga, 77-81, 1959.

Dorman, L. I, Iucci, N., and Villoresi, G.: The use of cosmic rays for continues monitoring and prediction of some dangerous phenomena for the Earth's civilization, Astrophysics and Space Science, 208, 55-68, 1993a.

Dorman, L. I., Iucci, N., and Villoresi, G.: Possible monitoring of space processes by cosmic rays, Proc. 23rd Intern. Cosmic Ray Conf., Calgary, 3, 695-698, 1993b.

Dorman, L. I., Iucci, N., and Villoresi, G.: Space dangerous phenomena and their possible prediction by cosmic rays, Proc. 23rd Intern. Cosmic Ray Conf., Calgary, 3, 699-702, 1993c.

Dorman, L. I., Iucci, N., and Villoresi, G.: The nature of cosmic ray Forbush-decrease and precursory effects, Proc. 24th Intern. Cosmic Ray Conf., Rome, 4, 892-895, 1995a.

Dorman, L. I., Villoresi, G., Belov, A. V., Eroshenko, E. A., Iucci, N., Yanke, V. G., Yudakhin, K. F., Bavassano, B., Ptitsyna, N. G., and Tyasto, M. I.: Cosmic-ray forecasting features for big Forbush-decreases, Nuclear Physics B, 49A, 136-144, 1995b.

Dorman, L. I., Iucci, N., and Villoresi, G.: Auto-model solution for non-stationary problem described the cosmic ray pre-increase effect and Forbush-decrease, Proc. of 25th Intern. Cosmic Ray Conf., Durban (South Africa), 1, 413-416, 1997.

Dorman, L. I., Iucci, N., Ptitsyna, N. G., and Villoresi, G.: Cosmic ray Forbush-decrease as indicators of space dangerous phenomenon and possible use of cosmic ray data for their prediction, Proc. 26th Intern. Cosmic Ray Conf., Salt Lake City, 6, 476-479, 1999.

Dorman, L. I., Belov, A. V., Eroshenko, E. A., Pustil'nik, L. A., Sternlieb, A., Yanke, V. G., and Zukerman, I. G.: Possible cosmic ray using for forecasting of major geomagnetic storms, accompanied by Forbush-effects, Proc. 28th Intern. Cosmic Ray Conf., Tsukuba (Japan), 6, 3553-3556, 2003.
Fenton, A. G., McCracken, K. G., Rose, D. C., and Wilson, B. G.: The onset times of Forbush-type cosmic ray intensity decreases, Canad. J. Phys., 37, 970-982, 1959.

Hruska, J. and Shea, M. A.: Solar-terrestrial predictions, Adv. Space Res., 13, 9, 451-455, 1993.

Kappenman, J. G. and Albertson, V. D.: Bracing for the geomagnetic storms, IEEE Spectrum, March 1990, 27-33, 1990.

König, H. and Ankermüller, F.: Naturwissenscahften, 17, 47, 486490, 1982.

McCracken, K. G. and Parsons, N. R.: Unusual Cosmic-Ray Intensity Fluctuations Observed at Southern Stations during 21-24 October 1957, Phys. Rev., 112, 5, 1798-1801, 1958.

McCracken, K. G., Rao, V. R., and Shea, M. A.: The trajectories of cosmic rays in a high degree simulation of the geomagnetic field, in: Technical report No. 77, Massachusetts Institute of Technology, USA, 1962.

McCracken, K. G., Rao, V. R., Fowler, B. C., Shea, M. A., and Smart, D. F.: Cosmic ray tables (asymptotic directions, variational coefficients and cut-off rigidities, in: IQSY Instruction Manuel, 10, London, 1965.

Munakata, K., Bieber, J. W., Yasue, S.-I., Kato, C., Koyama, M., Akahane, S., Fujimoto, K., Fujii, Z., Humble, J. E., and Duldig, M. L.: Precursors of geomagnetic storms observed by the muon detector network, J. Geophys. Res., 105(A12), 27 457-27468, 2000.

Nagashima, K., Sakakibara, S., Fujimoto, K., Tatsuoka, R., and Morishita, I.: Localized pits and peaks in Forbush decrease, associated with stratified structure of disturbed and undisturbed magnetic fields, Nuovo Cimento C, Serie 1, 13C, 551-587, 1990.

Ptitsyna, N. G., Villoresi, G., Dorman, L. I., Iucci, N., and Tyasto, M. I.: Natural and man-made low-frequency magnetic fields as a potential health hazard, UFN (Uspekhi Physicheskikh Nauk), 168, 7, 767-791, 1998.

Reiter, R.: Bio-meteorologie auf physikalischer Basis, Phys. Blätter, 11, 453-464, 1955.

Villoresi, G., Breus, T. K., Dorman, L. I., Iucci, N., and Rapoport, S. I.: The influence of geophysical and social effects on the incidences of clinically important pathologies (Moscow 1979-1981), Physica Medica, 10, 3, 79-91, 1994.

Villoresi, G., Dorman, L. I., Ptitsyna, N. G., Iucci, N., and Tyasto, M. I.: Forbush-decreases as indicators of health-hazardous geomagnetic storms, Proc. 24th Intern. Cosmic Ray Conf., Rome, 4, 1106-1109, 1995. 